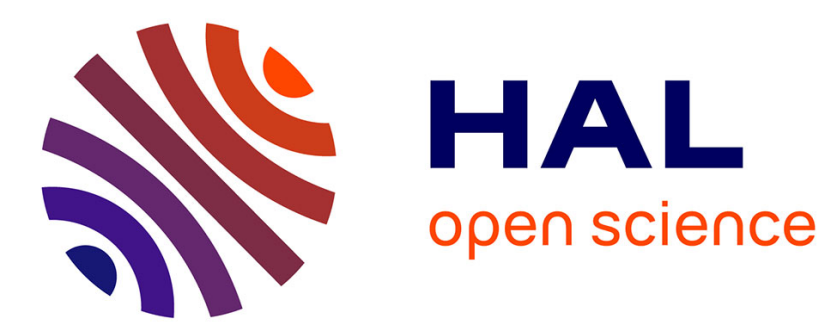

\title{
A programmable digital frequency synthesizer for a high resolution $\mathrm{nmr}$ spectrometer
}

\author{
S.K. Kan
}

\section{To cite this version:}

S.K. Kan. A programmable digital frequency synthesizer for a high resolution $\mathrm{nmr}$ spectrometer. Revue de Physique Appliquée, 1972, 7 (4), pp.329-333. 10.1051/rphysap:0197200704032900 . jpa00243635

\section{HAL Id: jpa-00243635 https://hal.science/jpa-00243635}

Submitted on 1 Jan 1972

HAL is a multi-disciplinary open access archive for the deposit and dissemination of scientific research documents, whether they are published or not. The documents may come from teaching and research institutions in France or abroad, or from public or private research centers.
L'archive ouverte pluridisciplinaire HAL, est destinée au dépôt et à la diffusion de documents scientifiques de niveau recherche, publiés ou non, émanant des établissements d'enseignement et de recherche français ou étrangers, des laboratoires publics ou privés. 
Classification

Physics Abstracts 05.20

\title{
A PROGRAMMABLE DIGITAL FREQUENCY SYNTHESIZER FOR A HIGH RESOLUTION NMR SPECTROMETER
}

\author{
S. K. KAN \\ Institut d'Electronique Fondamentale, Bâtiment 220, Université Paris-XI, 91-Orsay, France
}

(Reçu le 3 mai 1972)

\begin{abstract}
Résumé. - Nous décrivons un synthétiseur de phase et de fréquence utilisant des techniques digitales. Les phases, les fréquences et leur programmations sont commandées directement en logique TTL. Bien que cette réalisation ait été conçue pour la stabilisation, le découplage de spin et l'observation de raies en RMN, d'autres applications peuvent aisément être envisagées pour ce synthétiseur.
\end{abstract}

Abstract. - A low-cost filterless multi-signal frequency and phase synthesizer based on digital techniques is described. Usual functions such as frequency, phase selection and modulation are direct TTL programmable. Although it is built primarily for NMR field stabilisation, homonuclear spin decoupling and resonance line observation purposes, other applications in the frequency phase domain can also be envisaged.

Introduction. - In modern high resolution nuclear magnetic resonance (NMR) spectrometers, one usually needs three independent frequency sources respectively for field stabilisation, homonuclear spin decoupling and resonance spectrum observation purposes. These frequencies are normally derived either from three separate synthesizers or from a single unit associated with single sideband (SSB) generation. For example, H. S. Gutowsky et al. [1] described a hybrid system for their $40 \mathrm{MHz}$ spectrometer in which they use a $100 \mathrm{~Hz}$ to $31 \mathrm{MHz}$ Schomandl synthesizer to generate, by means of SSB techniques, frequencies of $40004 \mathrm{MHz}$ and $40000 \mathrm{MHz}(3.5 \leqslant f \leqslant 4.5)$ respectively for field stabilisation and line observation while the spin decoupling radio frequency (RF) field is obtained by harmonic generation of the synthesizer's output fundamental frequency. In both cases, at least one wide-range high frequency synthesizer is incorporated in the spectrometer.

In general, most, if not all of the high resolution NMR spectrometers' main fields $\left(H_{0}\right)$ are fixed and their sample coils tunable only over a limited frequency range. A wide-range high frequency synthesizer can be advantageously replaced by an audio frequency unit together with a HF high stability crystal controlled oscillator from which the desired frequency can be easily generated.

For our $240 \mathrm{MHz}$ high resolution NMR spectrometer, we have built three special AF synthesizers [2] and by this means have generated three high frequency sources using a SSB technique. This method requires, for each SSB signal, a pair of carrier waves in phase quadrature of frequency $f_{\mathrm{c}}(240 \mathrm{MHz})$ and another

\footnotetext{
* Laboratoire associé au CNRS.
}

pair of AF signals, also in phase quadrature, of frequency $f_{\mathrm{a}}(0-100 \mathrm{kHz})$. By multiplying and adding these signals with a balanced mixer and a hybrid $T$ junction, we can select either the upper-sideband $\left(f_{\mathrm{c}}+f_{\mathrm{a}}\right)$ or the lower-sideband $\left(f_{\mathrm{c}}-f_{\mathrm{a}}\right)$ signal with a good spectral purity. (Full details will be given in a future publication which will describe also time sharing, aperiodic synchronous detection etc. used in our system.)

In any of these three SSB generations, the relative phase of each pair of AF signals in phase quadrature must remain fixed and be independent of the frequency. Over a limited frequency range, however, satisfactory result can be obtained by means of phase shifting networks, but difficulty arises when we try to conserve the same phase shift over a wide range of frequencies as in our case. It is for this reason that we are led to the design and realisation of the synthesizer in which a system of frequency independent phase-shifting based on digital techniques is incorporated [3].

The phase-shifted signals so produced, in its simplest form, are triangular waves. These are used directly for our SSB generations. Although SSB signals are normally generated by means of AF sinewaves, in some special NMR applications, we can tolerate a spectrum of signals as long as the nearest unwanted sideband is frequency-separated far enough from the desired one. This is one of the reasons we have chosen the minimum value for $f_{\mathrm{a}}$ to be $10 \mathrm{kHz}$. (For applications that need sinewave ouptuts, a 50-word readonly memory is suggested as shown in figures 4,5 by which a sinewave reconstructed in 100 steps may be obtained (Fig. 8) [4].)

Two synchronous detectors are used respectively for line observation and field stabilisation purpose 


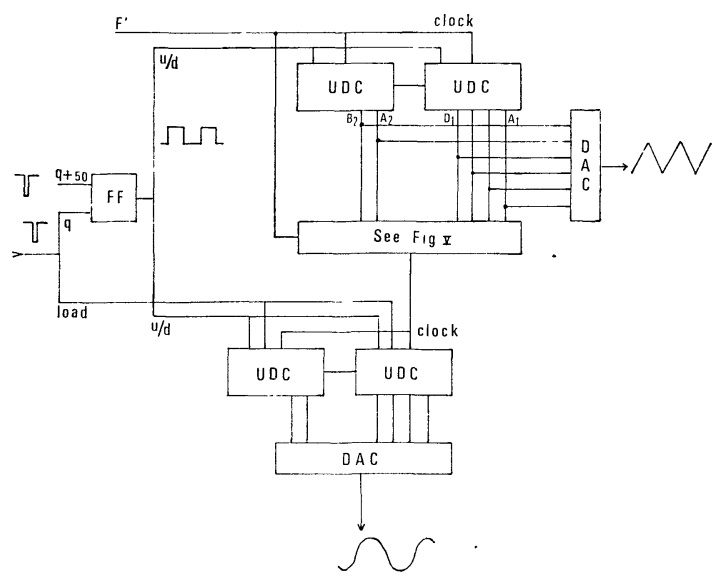

FIG. 4. - Sine wave output from a read-only memory.
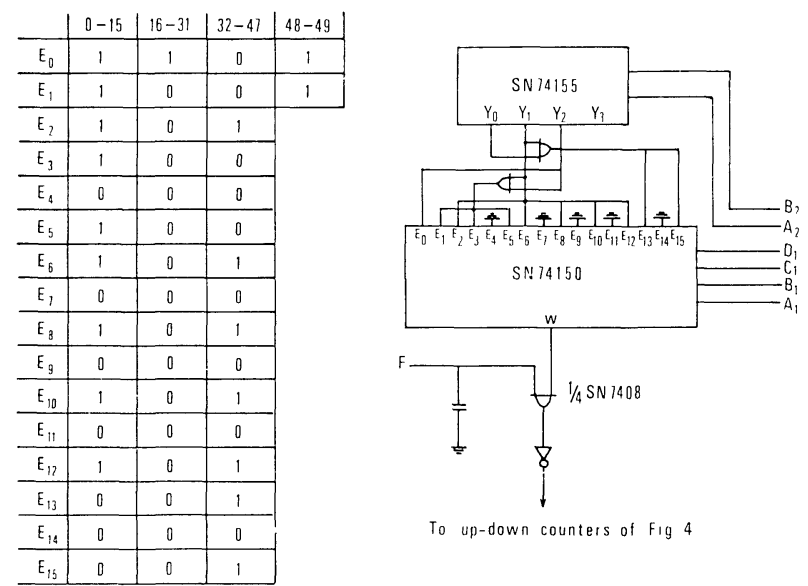

Fig. 5. - A 50-word read-only memory circuit.

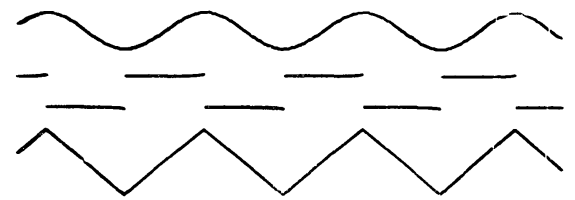

FIG. 8. - Sinewave obtained from a 50-word ROM.

in the spectrometer. The reference signals for these detectors are derived respectively from the AF signals used for SSB generations. In either case, it is a square wave whose relative phase shift can be varied over a range of 360 degrees, and also is independent of the operating frequency.

1. Basic principle. - The synthesizer we are going to describe is TTL compatible and the following functions are programmable either by BCD switches or control signals :

1) Frequency selection ;

2) Relative phase shift between sets of signals ;

3) Frequency and phase modulation.

Each set of signals is composed of one triangular wave, one square wave and two pulses displaced
180 degrees apart. It is generated by means of one rate multiplier per digit, two up-down counters, some logic gates and a digital to analog (D/A) converter.

As shown in figure 1 , a TTL signal from a very stable frequency source $(F)$ is applied to the clock

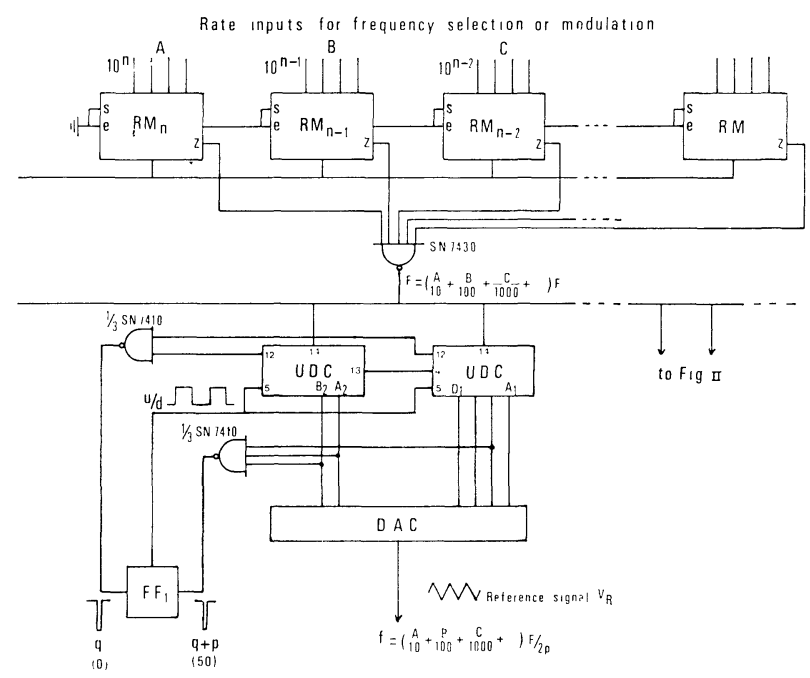

FIG. 1. - Basic circuit of the frequency synthesizer.

input of each Decade Rate Multiplier (RM) of the type SN 74167. In this diagram, they are connected from left to right in decreasing order of significant values. Each RM is enabled (e) and strobed (s) by that situated immediately to its left except the first one whose strobe and enable inputs are wired to ground.

Frequency selection is determined by the settings $A$, $B, C$ etc. of the $B C D$ switches (or corresponding TTL control signals) connected to the rate inputs of the multipliers.

According to the value of $A(0 \leqslant A \leqslant 9)$ indicated on the BCD switch, $\mathrm{RM}_{n}$ will deliver $A$ pulses at its output $(Z)$ for every 10 input pulses, the tenth is gated and appears on one of its output terminals by which $\mathrm{RM}_{n-1}$ is strobed and enabled. By so doing we thus obtain $B$ pulses from the $\mathrm{RM}_{n-1}$ output $(Z)$ for every 100 input pulses etc., etc.

These pulses from $Z$ are then summed by a logic gate (SN 7430) and the mean output frequency $F^{\prime}$ of which is now

$$
F^{\prime}=\left(\frac{A}{10}+\frac{B}{100}+\frac{C}{1000}+\cdots\right) F .
$$

This simple means allows us to obtain a very accurate ratio between $F$ and $F^{\prime}$. Unfortunately the pulses of frequency $F^{\prime}$ are normally unevenly spaced and direct application of it in the frequency synthesis process is rare. In our case, we divide $F^{\prime}$ by a number (100) sufficiently big to obtain acceptably regular triangular and square waves for field stabilisation and spin decoupling purposes since these two require a less stringent condition on the operating frequency (see 
later paragraph). In the case of the line observation signal, which is frequency swept, a perfect square wave for the reference channel of the synchronous detector is therefore imposed in order to assure a straight baseline. In a later paragraph, we will describe how this can be achieved by means of a special circuit.

In the present example, the output from the SN 7430 is applied to two up-down counters (UDC) (SN 74190 or SN 74191) connected in series to generate a triangular wave reconstructed in $2 p$ steps through the use of a digital to analog converter. This is done by letting the counters count up from a binary value of $q$ to $q+p$ and then down to $q$ to complete a cycle.

Values of $q$ and $q+p$ are decoded from the outputs of the counters by means of two Nand gates (SN 7420) the respective outputs of which form two pulses spaced 180 degrees apart. Pulse $q$ sets and pulse $q+p$ resets flip flop $F F_{1}$ from which the square wave is derived. The output from $F_{1}$ then controls the up-down counting process of the counters.

Thus by counting up and down and summing the weights of the six outputs $\left(A_{1}, B_{1}, C_{1}, D_{1} ; A_{2}, B_{2}\right)$ of the counters with a digital to analog converter (DAC), one obtains a triangular wave the frequency of which is given by

$$
f=\left(\frac{A}{10}+\frac{B}{100}+\cdots\right) \frac{F}{2 p} .
$$

From this equation, it is obvious that by choosing the ratio $F / 2 p$ equal to multiples of ten, the output frequency of the synthesizer is then given by the values indicated on the $B C D$ switches.

At the time of writing, the recommanded clock frequency for the rate multiplier is about $25 \mathrm{MHz}$ and for the up-down counters $20 \mathrm{MHz}$. Hence, we have chosen $F$ to be $10 \mathrm{MHz}$ and therefore $2 p=100$. The synthesizer's maximum frequency is then $10^{7} / 100=100 \mathrm{kHz}$.

2. Phase shifting. - The way by which the triangular wave is synthesized highly facilitates the means of obtaining relative phase shift between sets of signals. We shall only give one example to illustrate how it can be achieved digitally.

Phase shifting within a range of 0 to $2 \pi$ with respect to the reference signal $V_{\mathrm{R}}$ can be done using the data inputs of the up-down counters.

In order to obtain direct phase reading as indicated on the BCD switches, it is preferable to use decade up-down counters (DUDC) (SN 74190) for triangular wave generation (Fig. 2).

Relative phase shift is determined by the BCD switch settings $(m)$ connected to the data inputs of the counters. Synchronizing pulses $q$ and $q+p$ from the reference signal are used separately in order to cover the 4-quadrant phase shift. They are applied to an AND-OR-INVERT gate by which either pulse

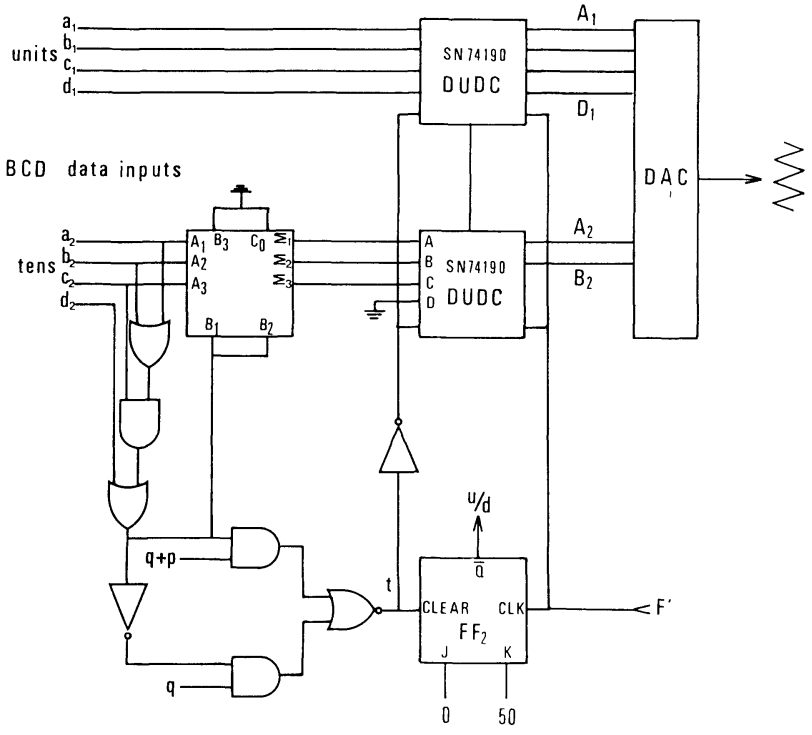

FIG. 2. - Phase selection or modulation using load inputs of Decade Up-Down Counters.

will be selected to appear at the output $(t)$ according to the state of a logic circuit. The output of the latter becomes high (logical 1) when the most significant digit $\left(\mathrm{a}_{2}, \mathrm{~b}_{2}, \mathrm{c}_{2}, \mathrm{~d}_{2}\right)$ of $m(0 \leqslant m \leqslant 99)$ is greater than 4 ; viz. $t=p$ for $0 \leqslant m \leqslant 49$, and $t=q+p$ for $50 \leqslant m \leqslant 99$. (A 4-bit Adder (SN 7483) is inserted for BCD to Bi-Quinary conversion.)

Pulse $t$ is connected to the «clear » input of flipflop $\mathrm{FF}_{2}$ and $\bar{t}$ the load input of the two counters. At the instant when this pulse occurs, the BCD data inputs are transferred to the output terminals of the counters which are also set to count «up » through the output $\bar{Q}$ of $\mathrm{FF}_{2}$. The initial values that appear across the terminals of the counters determine therefore the phase advance of $m 2 \pi / 2 p$ radians relative to $q$.

The rest of the process is identical to that described above and we then obtain a phase-shifted triangular wave from the output of the $\mathrm{D} / \mathrm{A}$ converter or a square wave from the $Q$ output of $\mathrm{FF}_{2}$.

If $m$ is fixed and equal to 25 as in our case, this signal will be in phase quadrature with the reference signal $V_{\mathrm{R}}(25.2 \pi / 2 p=\pi / 2)$.

3. Frequency modulation. - Frequency modulation can be realized by varying periodically the rate inputs of the rate multipliers. One form of internal modulation is the linear variation of the frequency. This is achieved by means of a series of decade up-down counters inserted between the rate inputs of the RM and the frequency - selection BCD switches as shown in figure 3. Two TTL signals of frequency $f_{\mathrm{dev}}$ and $f_{\text {mod }}$ are connected respectively to the clock input and the up-down control of each counter for this purpose. By setting the load inputs to 1 , the counters are then enabled and their outputs $A_{1} B_{1} C_{1} D_{1}$ etc. will vary in weights at a rate depending on $f_{\mathrm{dev}}$. According to 


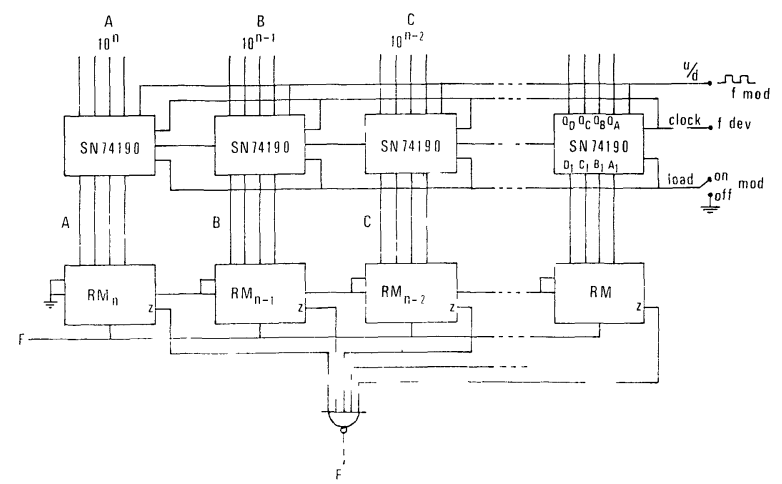

FIG. 3. - Frequency selection or modulation circuit.

the state of the up-down input of the counters, $f$, the output frequency of the synthesizer will be swept linearly up or down from its initial value (wobbulation). If the up-down control is a slow varying square wave of frequency $f_{\bmod }$, we will then obtain a frequency modulated output wave from the synthesizer.

Normal fixed output frequency results when the load inputs are set to 0 . Its value is then given by the readings on the $\mathrm{BCD}$ switches.

4. Phase modulation. - Phase modulation is obtained in a similar way to frequency modulation by varying periodically the data inputs of the up-down counters. Output waves from the D/A converters are thus modulated in phase relative to that of the reference signal.

5. Characteristics and applications of the three synthesizers. - In NMR line observation, the frequency must be known with great accuracy and must be wobbulable within a wide frequency range in order to explore the whole resonance line spectrum. Therefore we have chosen a $10 \mathrm{kHz}$ to $100 \mathrm{kHz}$ frequency band with a $0.01 \mathrm{~Hz}$ increment although the synthesizer itself has a $100 \mathrm{kHz}$ bandwidth. The choice of operating frequency range for line observation fixes that for the spin decoupling, which is variable in steps of $0.1 \mathrm{~Hz}$.

For ease of operation, we have also adopted the same frequency band of $1 \mathrm{kHz}$ resolution for the stabilisation field although it usually has a predetermined fixed value in most of the spectrometers with field stabilisation.

The following table summarizes the output signal wave forms and frequency resolution of each AF synthesizer used for SSB generations :

$F_{1}$ (Observation frequency)

Wobbulable between $10 \mathrm{kHz}$ to $100 \mathrm{kHz}$ in steps of $0.01 \mathrm{~Hz}$

2 triangular signals in phase quadrature.

1 square wave with variable phase shift in steps of $2 \pi / 100$ from 0 to $2 \pi$.
$\mathrm{F}_{2}$ (Stabilisation frequency)

Variable from $10 \mathrm{kHz}$ to $99 \mathrm{kHz}$ in steps of $1 \mathrm{kHz}$, Same as above.

$F_{3}$ (Decoupling frequency)

Variable from $10 \mathrm{kHz}$ to 2 triangular signals $100 \mathrm{kHz}$ in steps of $0.1 \mathrm{~Hz}\left\{\begin{array}{l}\text { in phase quadra- } \\ \text { ture. }\end{array}\right.$

The triangular wave created by summing 100 unevenly spaced pulses is slightly deformed. The additional harmonics due to this deformation have a negligible effect on the wanted sideband signal as mentioned above. On the other hand, the period of each triangular or square wave can vary up to a maximum of $3 \%$ from its mean value although its mean frequency is absolutely constant. This variation of the periodicity, at a slow rate, accounts for the modulation of the baseline during NMR spectrum recording. This is true when the resonance signal is detected with a synchronous detector. In the case of field stabilisation and spin decoupling, SSB signals generated with this sort of triangular wave can be directly applied to their respective circuit since the former has a resolution of $1 \mathrm{kHz}$ and the variation of the baseline level at the output of the synchronous detector can be easily filtered out. In the case of the latter (spin decoupling), the spin system plays the role of integrator and sees only the mean frequency.

In order to eliminate the defect on the triangular and square waves cited above, it is obvious that the easiest way to achieve this consists of applying a regular pulse train to the up-down counters for frequency and phase synthesis. The special circuit we propose is shown in figure 6 in which a Voltage

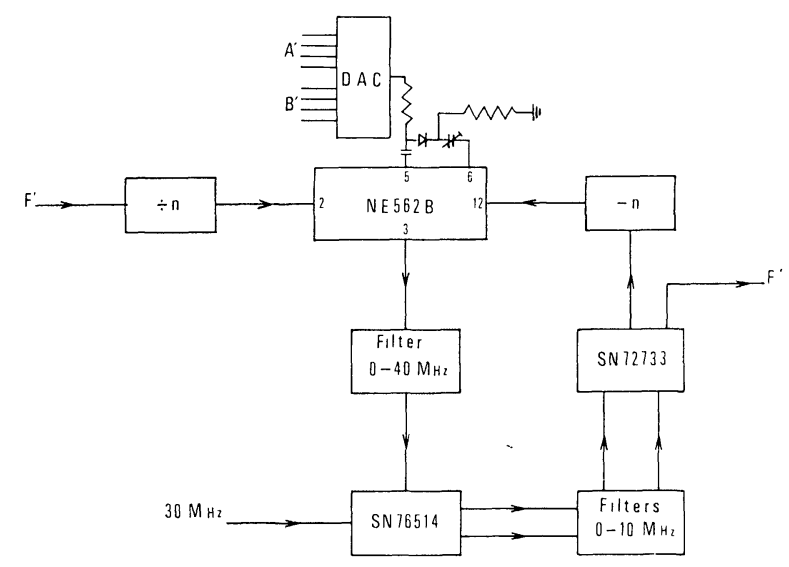

Fig. 6. - A voltage controlled oscillator for transforming unevenly spaced pulses into a regular wave train.

Controlled Oscillator (VCO) is phase-locked to the mean frequency $F^{\prime}$. The output voltage from the VCO, which is a regular wave of frequency $F^{\prime \prime}=F^{\prime}$, replaces the latter for triangular and square wave generations.

In this circuit, a frequency source of $30 \mathrm{MHz}$ 


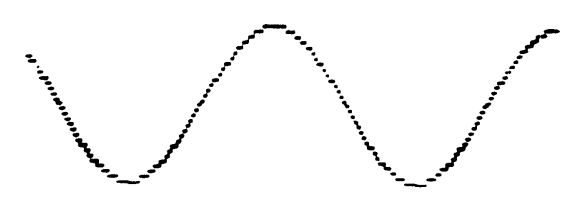

Fig. 7. - A group set of AF signals from the synthesizer.

derived from the $10 \mathrm{MHz}$ master oscillator is used. This is mixed with the output from the VCO (NE $562 \mathrm{~B}$ ) by means of a balanced mixer (SN 76514). The free oscillation frequency of the VCO can be varied from $30 \mathrm{MHz}$ to $40 \mathrm{MHz}$ by the use of a trimmer capacitor in series with a varicap (BB 105). The capacitance of the latter is directly controlled by a DC signal which is proportional to the instantaneous output frequency $(f)$ of the synthesizer.

The lower-sideband from the mixing is retained by a low pass filter and amplified by a differential amplifier (SN 72733). One of its outputs is compared with $F^{\prime}$ in order to phase-lock the system to constantly track the value of $F^{\prime}$ which varies from $1 \mathrm{MHz}$ to $10 \mathrm{MHz}$. Both signals are first divided by $n$ before returning to the phase comparator inputs of the VCO. This digital integration serves to transform $F^{\prime}$ into a low frequency square wave for more precise phase comparison purposes.

The other differential output $\left(F^{\prime \prime}\right)$ from the SN 72733, a regular wave train, now replaces $F^{\prime}$ for frequency and phase syntheses.

Acknowledgment. - The author tanks Dr Michel Sauzade, Director of Research of the Centre National de la Recherche Scientifique (CNRS) for his constant guidance and encouragement without which this work would never have been realized.

\section{References}

[1] Gutowsky et al. - « Frequency sweep, Field-Fre. quency stabilized, Double Resonance Spectrometer. ". The Review of Scientific Instruments, 1968, 39.

[2] Noordanus (J.). - « Frequency Synthesizers. A Survey of Techniques. » IEEE Transactions on Communication Technology, 1969, C-om17, 2.
[3] Kan (S. K.), Bloyet (D.). - « Programmable phaseshifter operates from dc to $100 \mathrm{kHz}$. » Electronic Design 1971, 19, 14.

[4] Parsons (B.). - «Binary rate Multipliers. » Texas Instruments, England, Recueil 1971.

Krausener (J.-M.). - «Multiplexeurs digitaux TTL SN 74150. » Texas Instruments France, Recueil 1971. 